\title{
Review: in older people, decline of cognitive function is more likely than improvement, but rate of change is very variable
}

Park HL, O'Connell JE, Thomson RG. A systematic review of cognitive decline in the general elderly population. Int J Geriatr Psychiatry 2003;18:1121-34.

\section{What is the rate of age related cognitive decline in the elderly population?}

\section{METHODS}

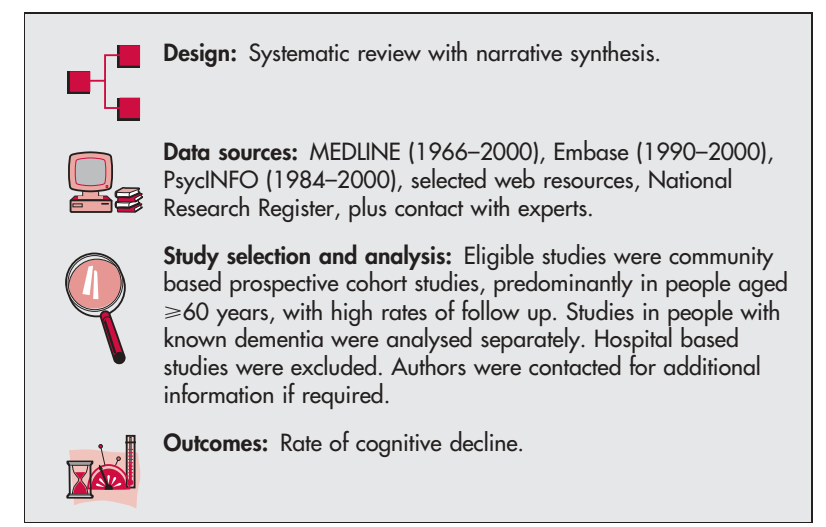

\section{MAIN RESULTS}

From 5990 possible abstracts, 19 papers met inclusion criteria. Metaanalysis was not possible due to heterogeneity of studies. It was not possible to determine the actual rate of age related cognitive decline in the general elderly population due to variations in testing, study design, and study reporting. The rates of cognitive decline varied among studies. In 12 studies, the annual decline in MMSE score ranged from $0.1-1.3$ points. In three studies, the annual decline in the Cam-Cog ranged from 0.96-1.6 points. All studies reported a relation between increasing age and some degree of cognitive impairment.

\section{CONCLUSIONS}

Cognitive decline is almost universal in the general elderly population and increases with age. The greatest declines are in the oldest subgroups. Good functioning at baseline is associated with smaller rates of decline. It was not possible to determine the actual rate of age related cognitive decline in the general elderly population due to variations in testing, study design, and study reporting. An

For correspondence: Helen Park, School of Population and Health Sciences, The Medical School, University of Newcastle, Newcastle upon Tyne, UK; H.L.Park@ncl.ac.uk

Sources of funding: the NHS Northern and Yorkshire Research and Development Directorate. international gold standard neuropsychological test battery is required to remedy this.

\section{NOTES}

Studies were of variable quality and used different types of assessments and outcomes. Quality was particularly poor for power calculations and reporting of the types of professionals carrying out neuropsychological testing. Loss to follow up in studies was high, ranging from $16.9 \%$ to $93.3 \%$.

\section{Commentary}

T he term "cognitive decline" (change in cognitive function over time) is increasingly used as an outcome for research in older populations because of deficiencies in the alternatives. "Dementia" represents an unsatisfactorily advanced outcome because, by definition, cognitive function has already declined to an extent where it is interfering with daily life. "Cognitive impairment" (relatively low performance on a cognitive test at a single assessment) potentially allows identification of earlier changes. However relative performance on cognitive tests may more strongly reflect sociocultural background than acquired deficits in later life. A difficulty with research in this area is the wide variety of cognitive assessments available that cannot easily be "combined" in systematic reviews. It is unfortunate that the most widely used assessment, the MMSE, was never designed for research of this sort and has limited sensitivity to change in the general population.

A major issue for research is the translation of results from the group to the individual. Epidemiological studies report findings averaged over large populations, whereas a clinician's focus is principally on the presenting individual. The results of this review article exemplify this difficulty. In particular it would be easy to misinterpret the abstract as implying that nearly all older people experience cognitive decline. What the authors instead are concluding is that the average change in cognitive function across an older population is generally negative. Variation within the samples was substantial. For example, the largest cited study using the MMSE (the Kungsholmen project) found a significant mean decline of 0.4 points per year. However the standard deviation (estimated from the quoted standard error) was 1.3 points. This implies that the range of change for about $95 \%$ of that sample lay between a decline of 3.0 points and an improvement of 2.2 points. Furthermore, anyone with experience of administering the MMSE will be aware that scores in a general population are clustered close to the maximum with less potential for improvement than decline. Cognitive decline may be frequently observed at a population level. However cognitive decline is by no means inevitable for individuals and this, surely, is an encouraging message for older people.

Robert Stewart, MD Institute of Psychiatry (Section of Epidemiology), London, UK 